Sergio Marotta. Profesor de Sociología del Derecho de la Facoltà di Scienze della Formazione de la Università degli Studi "Suor Orsola Benincasa”, Napoli. Sus principales intereses científicos atañen a la transformación de los ordenamientos jurídicos de la globalización. Sus trabajos recientes son L'individuo senza Stato. Globalizzazione e sfera pubblica Editoriale Scientifica, Napoli, 2008; "La depubblicizzazione dei servizi idrici dalla municipalizzazione all'obbligo di esternalizzazione", en Мunus, no. 1, 2011; "La via italiana ai beni comuni", en Aedon, 1, 2013; “On The Critical Relationship Between Citizenship and Governance: The Case of Water Management in Italy", en Urbanities, vol. 4, 2, November 2014.

Contacto: sergio.marotta@unisob.na.it 


\section{EL DERECHO DE LA CRISIS ENTRE JUSTICIA SOCIAL Y JUSTICIA DEL MERCADO}

\section{Sergio Marotta}

Università degli Studi “Suor Orsola Benincasa”, Napoli

\section{CRISIS LAW BETWEEN SOCIAL AND MARKET JUSTICE}

DOI: $10.17450 / 150208$

Fecha de recepción 30 de septiembre 2015; fecha de aceptación 22 de octubre 2015. El artículo es fruto de un proyecto de investigación desarrollado en la Facoltà di Scienze della Formazione - Università degli Studi “Suor Orsola Benincasa”, Napoli.

\section{Resumen}

El artículo se basa en la compleja relación entre derecho y economía, destacando tres pronunciaciones de la Corte Constitucional italiana. El papel de la justicia constitucional en la reglamentación social en un contexto de grave crisis económica involucra la naturaleza de la racionalidad que orienta estas pronunciaciones del Juez de las leyes. Se comparan racionalidad económica y racionalidad jurídica por medio del filtro de dos perspectivas teóricas diferentes. El aparato teubneriano se vuelve analizar para intentar garantizarle al derecho el control de las tendencias expansivas de la racionalidad económica. La oscilación de la jurisprudencia constitucional entre las dos racionalidades y los 
cambios de paradigma interpretativo impuesto con la modificación de la misma Carta fundamental con el nuevo art. 81, parecen dificultar esta solución. De ahí que parezca necesario recuperar la diferente perspectiva propuesta por el análisis foucaultiano, porque por medio de ésta se puede comprender la dimensión de poder en la que se realiza el proceso de veridición contextual a la racionalidad económica.

\section{Palabras clave}

Derecho, economía, Teubner, Foucault, jurisprudencia constitucional.

\section{Abstract}

This essay addresses the complex relationship between law and economy, using as exemplar cases three rulings made by the Italian Constitutional Court. In the context of the economic crisis, the role played by constitutional jurisprudence in ruling social matters, queries the rationality that informs certain 'Law judgement' sentences. The discussion compares economic and legal rationality through two different theoretical perspectives. Teubner's point of view is revisited looking at how the law may control the expansion trend of economic rationality. Constitutional ruling fluctuates between these two rationalities, and the interpretative paradigm changes as a consequence of changes in the Costitution (see, for example, the new art. 81 of the Italian Constitution). These factors cast doubt on Teubner's perspective. On the other hand, Foucault's paradigm helps us to understand power as a form of "truth telling" that attends economic rationality.

\section{Keywords}

Law, Economy, Teubner, Foucault, Constitutional Jurisprudence.

\section{Premisa}

Algunas sentencias recientes de la Corte Constitucional italiana nos hacen reflexionar acerca del conflicto actual entre las políticas de austeridad puestas en marcha en los países con grave deuda pública y la defensa de los derechos fundamentales que, por lo general, están relacionados con el ámbito patrimonial. La reforma consti- 
tucional de 2012, derivada de la adhesión italiana al fiscal compact ${ }^{1}$, ha modificado el art. 81 de la Constitución, deteniéndose sobre todo en la necesidad de hacer cuadrar el balance y agudizando el choque entre un legislador que quiere recuperar recursos para salir de la crisis y la Corte Constitucional, cuya finalidad es defender el derecho a una retribución que lleve a una existencia libre y digna, a la tutela de los derechos sindicales, a una tasación ecuánime y progresiva basada en una diferente capacidad contributiva.

Las tres sentencias que vamos a examinar a lo largo de este trabajo contribuyen significativamente a aclarar los motivos del choque entre Legislador y Juez de las leyes. En particular, con la primera se declaró la ilegitimidad constitucional de la disciplina que en 2008 había introducido en el ordenamiento italiano un impuesto adicional sobre la renta de las sociedades (IRES) a cargo de las empresas comerciales del sector energético, llamado Robin Hood Tax. Con la segunda, se declaró la ilegitimidad constitucional de la disciplina que, en 2011, había negado el reajuste al coste de la vida, relativo a los años 2012 y 2013, de las pensiones que superaban tres veces las mínimas. Además, con la tercera se declaró la ilegitimidad constitucional del bloque de la contratación en la función pública que había implicado un ahorro para el tesoro del Estado en detrimento de los derechos patrimoniales y sindicales de los trabajadores del sector público.

Partimos de estas tres sentencias para reflexionar acerca de la clásica función distributiva del derecho, alternativa con respecto a la de la economía y, por lo general, acerca de las relaciones entre economía y derecho, mejor dicho entre racionalidad económica y racionalidad jurídica, tal como se han ido estructurando en los contenidos y en los efectos de la legislación de la austeridad, que en Italia fue introducida tras la crisis financiera global de 2008.

Además, en este artículo nos proponemos individuar el papel real de la justicia constitucional en la reglamentación social y, sobre todo, en relación con la interpretación en términos de constitución material (según lo establecido por el neoconstitucionalismo) ${ }^{2}$ del derecho de la crisis, intentando individuar algunas tendencias de la evolución del

\footnotetext{
1. Es notorio que el tratado acerca del Fiscal compact preveía que en los ordenamientos de los Estados miembros se insertara el principio del equilibrio presupuestario con disposiciones preferiblemente constitucionales. Otros Estados insertaron este principio en su ordenamiento gracias a leyes orgánicas (por ejemplo, España y Francia) por no considerarse vinculadas a la positivización del principio por medio de disposiciones de tipo constitucional (cfr. la decision n. 653 de 2012 del Conseil constitutionnel francés). En cambio, Italia -que en aquel entonces estaba colapsando por la crisis deudora- quiso incluir este principio en su ordenamiento con una reforma constitucional.

2. El neoconstitucionalismo no identifica a la Constitución material "con principios que expresan una situación determinada por la fuerza, sino con la moral social, entendida como un conjunto difundido y compartido de principios de justicia” (M. Dogliani, "Costituzione in senso formale, materiale, strutturale e funzionale. A proposito di una riflessione di Gunther Teubner sulle tendenze autodistruttive dei sistemi sociali”, en Diritto Pubblico, 2, 2009, pp. 295-315: 295).
} 
sistema jurídico italiano y de las relaciones entre decisor político y juez que en el actual contexto histórico parecen particularmente significativas ${ }^{3}$.

De ahí que los instrumentos teórico-interpretativos que se adoptan en este artículo sean el funcionalismo teubneriano en sus desarrollos más recientes y las clásicas consideraciones foucaultianas sobre el neoliberalismo de sus clases en el Collège de France de 1978-1979 acerca del nacimiento de la biopolítica.

Por lo general, llegaremos a la formación de una nueva disciplina constitucional sobre el balance de cuentas como síntoma de la crisis profunda del Welfare State que desemboca en la 'tiranía' de la racionalidad económica también en sectores tradicionalmente sometidos a la reglamentación estatal de la economía.

\section{Las sentencias de la Corte Constitucional italiana}

En la primera sentencia, la n. 10 del 11 de febrero de 2015 sobre la llamada Robin Hood Tax, por la violación del principio de igualdad establecido por el art. 3 y de aquel sobre la capacidad contributiva establecido por el art. 53 de la Constitución, la Corte Constitucional ha declarado ilegítima la norma que imponía un impuesto adicional sobre la renta Ires de las sociedades que trabajaban en el sector del comercio de los productos petrolíferos y de sus derivados y que ganaban más de 25 millones de euros. Este impuesto adicional fue introducido por el Gobierno con el Decreto Ley n. 112/2008, dependiendo de los niveles excepcionales que las cotizaciones de los productos energéticos habían alcanzado durante el verano de 2008 y se quedó en el ordenamiento tributario por las condiciones de emergencia económica que se dieron tras la crisis de aquel mismo año ${ }^{4}$.

\footnotetext{
3. Tal como ha afirmado recientemente Alberto Febbrajo recurriendo a una obra de Niklas Luhmann de 1993, "la Corte constitucional puede ser considerada como el órgano más político del sistema judicial y el órgano más judicial del sistema político. Ella no solo está vinculada al sistema jurídico y al sistema político, sino también tiende a realizar funciones complejas de selección, estabilización y variación, encomendando su actualización a una especie de 'diálogo constitucional' que ya actúa a nivel interestatal y, en los relativos ámbitos de competencias, no solo está afectado por otros sistemas jurídicos sino también por sistemas no jurídicos como aquellos de la economía o de la religión” (A. Febbrajo, "Dal diritto riflessivo al diritto frammentato", en A. Febbrajo y F. Gambino (eds.), Il diritto frammentato, Giuffré, Milano, 2013, pp. 167-213: 191). 4. Entre los muchos comentarios a la n. 10 del 11 de febrero de 2015, cfr. L. Sica, "Genesi, applicazione e dichiarazione di incostituzionalità della Robin Hood Tax. Note a margine di alcune mutazioni”, en Diritto Pubblico, 2, 2015. Sobre el argumento, véase también E. De Mita, "Sulla Robin tax una bocciatura assai discutibile", en il Sole 24 ore, 8 de marzo de 2015; A. Morelli, "Tutela nel sistema o tutela del sistema? Il caso come occasione della tutela sistemica della legalità costituzionale e la forza politica del giudice delle leggi (notazioni a margine di Corte cost. n. 10/2015)", en Forum di quaderni costituzionali, 27 de abril de 2015; I. Massa Pinto, "La sentenza della Corte costituzionale n.10 del 2015 tra irragionevolezza come conflitto logico interno alla legge e irragionevolezza come eccessivo sacrificio di un principio costituzionale: ancora un caso di ipergiurisdizionalismo costituzionale", en Costituzionalismo.it, 1, 2015.
} 
Lo más interesante para el análisis de la relación entre derecho y economía es la decisión de la Corte de calcular los efectos de la declaración de ilegitimidad constitucional desde el día siguiente a la publicación en la Gaceta Oficial. Esta decisión atañe a consideraciones exclusivamente económicas dependiendo de la influencia de la decisión de los jueces en el presupuesto nacional en el caso de aplicación del normal efecto retroactivo que, entre otras cosas, para la Corte llevaría a una redistribución irrazonable de la riqueza porque, a pesar de la prohibición del legislador, el impuesto influía en el precio pagado por los consumidores. La norma es ilegítima, pero una declaración de incostitucionalidad impondría al Gobierno y al Parlamento una maniobra presupuestaria que incluso pondría en peligro los principios de igualdad y de solidaridad social.

Además, recurriendo a una jurisprudencia anterior, la Corte recuerda que su tarea institucional exige que la Constitución sea garantizada como un conjunto unitario, para asegurar "una tutela sistémica y no fraccionada" (sentencia n. 264 de 2012) de todos sus ciudadanos y principios implicados en la decisión", so pena de la tiranía de uno de sus derechos sobre los demás.

La segunda sentencia, la n. 70 del 30 de abril de 2015, decidió la ilegitimidad constitucional del bloqueo de la revaluación conforme al coste de la vida para 2012 y 2013 de las pensiones superiores a tres veces las mínimas. También en este caso se trata de una medida urgente de la que se hizo cargo el gobierno en 2011 con el Decreto Ley del 6 de diciembre de 2011, n. 201, justificado por el decisor político en la base de la situación financiera contingente y de la necesidad de no empeorar ulteriormente el déficit del presupuesto nacional.

La Corte aclara que la medida de urgencia del Gobierno es "un acto de autoridad de carácter ablatorio, con el objetivo de recaudar recursos para la Hacienda" que, además, se diferencia del pasado, cuando el legislador solo perjudicó las pensiones más elevadas, y declara que la norma que impide la revaluación es constitucionalmente ilegítima puesto que el mecanismo de revaluación automática de jubilación "se propone tutelar los procedimientos de jubilación de la depreciación monetaria, que tiende a afectar a las prestaciones de previsión también cuando no hay inflación"5.

A diferencia del caso anterior, la Corte no quiere limitar los efectos retroactivos de su sentencia, y restituye a los jubilados lo que no habían pagado en los años 2012 y 2013. Esta sentencia fue contrastada por todos los que acusaron la Corte de comprometer las finanzas públicas con otros gastos, sustrayendo recursos al poder discrecional del

$\overline{\text { 5. Corte Const. } 30}$ de abril de 2015, n. 70, punto 5 de las Consideraciones de derecho. 
Gobierno a la hora de poner en marcha la maniobra presupuestaria ${ }^{6}$. Frente a esta necesidad el Gobierno formuló el Decreto Ley del 21 de mayo de 2015, n. 65, imaginando el derecho al reembolso en un sistema de sumas fijas -en realidad, bastante bajas- que aumentan a medida que aumente la cantidad de dinero subyacente que se va reintegrando.

Además de otros aspectos de la decisión menos importantes para este trabajo, es suficiente recordar que ella se basa en el criterio de proporcionalidad respecto de la cantidad y cualidad del trabajo prestado (art. 36, primer apartado, Const.), en el criterio de conveniencia (art. 38, segundo apartado, Const.) de la prestación previsional y en la obligación para el legislador de respetar las exigencias mínimas de protección de la persona y de tutela de los trabajadores.

La tercera sentencia atañe a otra intervención del legislador que quiere disminuir los derechos de los trabajadores del sector público, una vez más justificada por la difícil situación económica y financiera. En particular, la Corte ha considerado que era constitucionalmente legítimo introducir un sistema de medidas para limitar los derechos patrimoniales y no patrimoniales, relacionados con la dinámica sindical de los trabajadores de la función pública para tutelar el interés superior del buen funcionamiento de las cuentas públicas. Así que la situación económica contingente justifica la congelación de los sueldos para los dependientes públicos que el Gobierno y el Parlamento han decidido poner en marcha también a raíz de la nueva formulación del art. 81 de la Constitución. Así que la Corte no reconoce en las disposiciones de ley a examinar el perjuicio a una retribución que asegure una vida libre y digna a la familia y a los propios dependientes públicos, sobre todo si pensamos en que la dinámica retributiva pública funcionaba mucho mejor que la de los sectores privados de la economía.

Congelar los sueldos puede ser legítimo solo en ocasiones excepcionales; sin embargo, para la Corte no lo es congelar por mucho tiempo la contratación colectiva en la función pública. Así que si no es inconstitucional bajo el punto de vista de la violación del art. 36 Const., la norma examinada por la Corte se puede censurar bajo el punto de vista de la violación del derecho a la libertad sindical. Este derecho puede ser reducido por exigencias superiores -como el buen funcionamiento de las cuentas públicas- pero solo por períodos limitados. De ahí que el bloqueo de la contratación decidido por el gobierno se considere inconstitucional porque tiende a prolongar el bloqueo por demasiado tiempo, implicando una sistematicidad de la suspensión que desemboca "en un equilibrio irrazonable entre libertad sindical [...] y exigencias de distribución racional

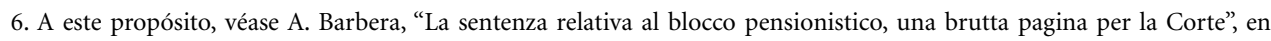
Rivista Aic, 15 de mayo de 2015. 
de los recursos y control del gasto, en una coherente programación financiera (art. 81, primer apartado, Const.)" ${ }^{\prime \prime}$.

En estas tres sentencias el Juez constitucional interviene sobre medidas sugeridas por un legislador cuya única finalidad es cobrar rápidamente reduciendo el gasto público para evitar el estándar de las finanzas públicas sin evaluar las consecuencias de las medidas sobre los destinatarios en la base de los principios constitucionales.

Si en el primer caso las medidas del Gobierno chocan, por un lado, con el principio del libre mercado y, por otro lado, con el de la progresividad impositiva, en el segundo se contraponen con los derechos de los trabajadores a tener una pensión adecuada a las cantidades y cualidades del trabajo desarrollado, mientras que en el tercero las medidas que preveen la disminución del gasto para los sueldos públicos se contraponen con los derechos sindicales de los trabajadores a las negociaciones colectivas.

Por lo que a la Robin Hood Tax se refiere, el legislador tiene motivaciones redistributivas: puesto que ella debe cobrar dinero, el gobierno quiere afectar a una categoría de contribuyentes que, en un período de crisis generalizada, había guardado y quizá aumentado sus ganancias, pues su capacidad contributiva, porque la demanda de productos petrolíferos es rígida y sigue también en momentos de cris ${ }^{8}$. Negando la constitucionalidad de la norma, la Corte no aprueba la labor del decisor político, pero determinando la irretroactividad de la declaración de inconstitucionalidad parece atenerse al vínculo presupuestario establecido por el art. 81: si se tuviera que devolver el dinero pagado por los petroleros, incluso se debería realizar una nueva maniobra financiera con efectos devastadores en el presupuesto público.

La segunda sentencia afecta a una medida que apunta a cobrar dinero, aunque en este caso la Corte, considerando la lesión del derecho de las personas a una retribución diferenciada capaz de asegurarles una vida digna, no emplea el principio de la irretroactividad de la declaración de inconstitucionalidad y obliga el decisor político a intervenir para restablecer el respeto de los derechos de los jubilados. La siguiente intervención del gobierno solo parcialmente hace caso a las indicaciones de la Corte, considerada la exigüidad de las sumas pagadas.

7. Corte Const. 23 de julio de 2015, n. 178, punto 17 de las Consideraciones de derecho.

8. Si las argumentaciones del legislador y sobre todo del ministro Giulio Tremonti, que primero había celebrado la iniciativa, fueron aceptadas muy bien desde el punto de vista redistributivo, algo diferente le tocó a la dinámica aplicativa de la disciplina que estamos analizando. A esta le siguió una prohibición de traslación que no se podía penalizar de ninguna manera, mientras el tributo fue transferido de las empresas tasadas a los consumidores finales, gracias al aumento de los precios de los productos. Este acontecimiento se reconstruye perfectamente en los muchos informes anuales sobre el tema de la Autoridad de la energía eléctrica, del gas y del sistema hídrico. Estos informes se hallan en el sitio institucional de la Autoridad (http://www.autorita.energia.it/it/index.htm). 
La tercera sentencia atañe a otra intervención del legislador para reducir los derechos de los trabajadores del sector público, otra vez legitimada por la particular gravedad de la situación económica y financiera. En este caso, la Corte acepta el sacrificio económico de los trabajadores por la difícil situación financiera, aunque considere inconstitucional el largo bloqueo de la contratación colectiva tanto en la parte retributiva como en la normativa, sobre todo si este bloqueo va a durar mucho tiempo.

Por lo general, las sentencias examinadas impulsan la Corte constitucional a aflojar la bolsa del gasto público, a pesar de las medidas anticrisis de la temporada anterior, pues entre 2008 y 2012.

La relación entre Corte constitucional y gasto público siempre ha sido bastante atormentada en la vida constitucional italiana, pues la Corte muchas veces ha aumentado la cantidad del gasto a través de las sentencias aditivas. Tanto en la experiencia anterior como en la más reciente, el fenómeno se considera bastante problemático, porque rompe el circuito entre decisión de gasto y mecanismo democrático representativo que -gracias al principio de autoimposición- se halla en el modelo contractualista dominante en la descripción del funcionamiento y de las razones de la forma del Estado democrático. La idea de que algunos gastos se convierten en constitucionalmente obligatorios ya sea directamente, porque son necesarios a la tutela de derechos constitucionalmente tutelados, ya sea indirectamente, porque son discriminatoriamente omitidos por el legislador respecto de prestaciones previstas, en cambio, para situaciones similares, ha sido un tema que desde siempre ha complicado los confines entre la función de la justicia constitucional y la del poder discrecional legislativo?.

\section{La asignación de los recursos entre derecho y mercado}

La crisis financiera de 2008 y las políticas de austeridad y de reducción del gasto público realizadas por cada Estado nacional para salvar el equilibrio del presupuesto nacional nos llevan a reflexionar sobre el uso de recursos colectivos que ya no parecen inagotables: se vuelve a hablar del tema de la asignación, mejor dicho los criterios de distribución de bienes y recursos escasos.

En la primera modernidad ${ }^{10}$, caracterizada por la presencia de Estados y economías nacionales, la función de distribución de bienes y recursos escasos se desarrollaba en los

9. Véanse, entre otros, las actas del seminario que se dictó sobre la Corte Constitucional, titulado "Le sentenze della Corte costituzionale e l'art. 81 u.c. della Costituzione", el 8 y el 9 de noviembre de 1991.

10. Hablo de primera modernidad refiriéndome a Ulrich Beck (Cfr. U. Beck, Was ist Globalisierung, Suhrkamp, Frankfurt, 1997). 
mercados gracias a las leyes de la economía, es decir, a la ciencia que estudia específicamente la asignación de recursos limitados entre usos alternativos para maximizar la riqueza producida.

A la función distributiva basada en las reglas de la economía se le añadía, para contenerla y equilibrarla al mismo tiempo, la presencia de ordenamientos estatales más o menos amplios desde el punto de vista territorial que desarrollaban funciones distributivas y que, a su vez, redistribuían la riqueza producida.

Hacia mitad de los años setenta, el sociólogo del derecho Lawrence M. Friedman acertó diciendo que "Legal decisions are by their very nature economic. They allocate scarce goods and services. The legal system is in this sense a rationing system. [...] Every function of the law, general or specific, is allocative" ${ }^{11}$.

Desde el punto de vista de la ciencia económica, ordenamientos jurídicos estatales y mercados lograron coexistir porque se opinaba que, en realidad, los Estados nacionales eran un modelo organizativo y de gestión de tipo exclusivamente transitorio, funcional a las exigencias de los mercados ${ }^{12}$. El hecho de que la dimensión estatal de los mercados sea transitoria es una especie de principio implícito en la regla económica que impone la maximización de las ganancias: tan pronto como el desarrollo tecnológico, la velocidad y cualidad de los transportes, los tipos de organización del trabajo y de la producción los hubieran posibilitado, los mercados se liberarían de los límites de las normas estatales y sobrepasarían los confines nacionales.

En los espacios deslocalizados y en los confines administrativos de los Estados nacionales, la separación entre derecho y economía se dio artificialmente por la coexistencia de dos criterios de asignación diferentes: el primero debido a la racionalidad económica y el segundo a la racionalidad política interpretada por medio de normas del ordenamiento jurídico de fuente estatal.

La coexistencia de estos dos tipos de función distributiva duró varios siglos porque, en la esfera económica, el derecho estatal se limita a la mera garantía de las transacciones que ocurren en el mercado que, de forma autónoma, "sets prices and disposes of goods and services through private agreements", mientras el derecho mismo "sustains, defines, and limits the area in which the free markets operate"13.

11. L. M. Friedman, The Legal System. A Social Science Perspective, Russell Sage Foundation, New York, 1975, p. 20 12. Cfr. K. Ohmae, End of Nation State. The rise of regional economies, Harper Collins Pubblisher, Berkeley, 1995. 13. L. M. Friedman, The Legal System, p. 21. 
Volviendo a lo del derecho, podemos decir que cuando los estudiosos perdieron la confianza en la que, para todo un siglo, había sido la teoría dominante, gracias a la cual el derecho se pudo considerar un sistema universal y completo, los juristas empezaron a dirigir su interés hacia la economía, por ser entre todas las demás ciencias sociales el candidato que supo ofrecer mejores y correctas soluciones a los problemas jurídicos ${ }^{14}$. De tal forma que la economía ofreció al derecho los contenidos que las normas jurídicas, siendo reglas de por sí neutras, habían sacado constantemente de otras esferas de la sociedad, como la política o la esfera afectivo-familiar, o de intereses diferentes de los económicos.

Francesco Galgano ha dicho que "derecho y economía se separan con la llegada de la Ilustración y de la Revolución Industrial. La primera lleva a la proclamación de las libertades burguesas, entre las que se hallan la libertad de comercio y de industria; la segunda lleva a la primacía del capital industrial respecto del comercial"15. Así que en su opinión aquella entre derecho y economía fue desde el principio una separación artificial que ha ido profundizándose con la progresiva construcción de los Estados nacionales y con la afirmación del monopolio de la producción legítima de las normas por parte del Estado. Finalmente, la separación entre economía y derecho se ha perfeccionado con el cierre de las fuentes formales del derecho, cuando "ningún Estado reconoce ningún otro derecho menos aquel que él mismo ha prescrito; las costumbres comerciales retroceden al último nivel en la jerarquía de las fuentes. Se da una contradicción creciente entre el comercio, que se extiende cada vez más a ámbitos internacionales, y el derecho que, por lo contrario, disminuye y se fragmenta en específicas unidades estatales"16.

También el economista Alessandro Roncaglia está convencido de que la separación entre derecho y economía ha aumentado a caballo de los siglos XVII y XVIII, y de que se ha empezado a distinguir la libertad económica de las demás libertades 'políticas'. Él considera la distinción entre libertad económica y las demás libertades como resultado del pensamiento reaccionario sucesivo a la Revolución francesa, pues cuando las monarquías europeas lograrían eliminar las demás libertades, dejándole al individuo la libertad de perseguir sus intereses económicos. La libertad económica sería la única posible por ser la única legítima para los gobiernos reaccionarios sucesivos a la Revolución francesa y a la época napoleónica.

14. Cfr. G. Minda, Postmodern Legal Movements: Law and Jurisprudence at Century's End, New York University Press, New York, 1995.

15. F. Galgano, La globalizzazione nello specchio del diritto, il Mulino, Bologna, 2005, p. 46.

16. Ibid., p. 47. 
En la economía clásica y sobre todo en el pensamiento de Adam Smith no habría ninguna distinción entre libertad económica y libertad política, pues ellas coinciden respetando la autonomía decisional del ciudadano ("Recordemos el célebre pasaje de la Teoría de los sentimientos morales: 'Está claro que todo hombre, desde todos los puntos de vista, sabe cuidarse más que si lo hicieran los demás””). Para Roncaglia, los intérpretes de Smith "durante la reacción conservadora inglesa frente al Terror jacobino" consideraron oportuno separar los dos aspectos: "la libertad en ámbito económico a la que se seguía aspirando, y la libertad en ámbito político, que se arrinconaba prudentemente quizás en la espera de tiempos mejores"17.

Sin embargo, esta distinción desencadenó muchas consecuencias teóricas, porque al formular la tesis originaria de Smith se volvió a considerar el Estado como "un enemigo del mercado -en lugar de un complemento esencial a su buen funcionamiento" y, por consiguiente, las reglas impuestas por la autoridad estatal han sido consideradas como “lazos y lazuelos' que refrenan la libertad de acción individual”18.

La crisis financiera global de 2008 comprimió la función distributiva desempeñada por el derecho respecto de la economía, y puso la libertad económica en el centro de la libertad política, volviendo a emprender el camino que, de alguna manera, había sido interrumpido por la paréntesis de los Estados nacionales con propios ordenamientos autónomos.

Pero ya a finales del siglo pasado se asistió a la rápida difusión entre los estudiosos del derecho de teorías inspiradas en la primacía de la economía sobre el derecho. Piénsese en la corriente de estudios llamada Law and Economics (que, al principio, consistía en la idea de que "el derecho fuera economía y de que la economía fuera una ciencia de la 'razón' neutral y apolítica” $\left.{ }^{19}\right)$, que implicó algunas secuelas que hace algunos años describió muy bien Alain Supiot: "Where Marx Failed, the Law and Economics movement might well be succeeding, winning jurist over to accepting the importance of setting law back on its 'real' feet, that is, on its economic base" 20.

Mientras el derecho estatal pretendería imponer sus reglas generales y abstractas también al ámbito de la economía, eliminando las reglas del mercado de las fuentes formales o, mejor dicho, considerándolas solo una especie de extrema ratio allí donde el derecho estatal no fuera suficiente para reglamentar los intereses en juego, con la globalización y con la siguiente crisis de 2008 se asiste a un proceso en el que el derecho

17. A. Roncaglia, Il mito della mano invisibile, Laterza, Roma-Bari, 2005, p. 69.

18. Ibid., pp. 69 s.

19. G. Minda. Postmodern Legal Movements, pp. 83 y ss.

20. A. Supiot, Homo juridicus. On the Anthropological Function of the Law (2005), Verso, London- New York, 2007, p. 85 
sucumbe ante las reglas del mercado. Además, cuando debe intervenir en la economía para defender posiciones residuales, o bien porque lo obligan las presiones de las clases más débiles, debe emplear necesariamente las reglas del derecho privado y comercial comunes a todos los demás sujetos del mercado. Solo renunciando a todo privilegio relacionado con la antigua concepción de su naturaleza, el derecho "no altera la paridad de la competencia económica y no introduce en el mercado elementos de anomalía y singularidad"21.

\section{Teubner y Foucault}

Con ocasión de la inauguración en Nápoles del año académico del Istituto Italiano per gli Studi Storici, Günther Teubner subrayó que hoy debemos afrontar el problema del desplazamiento de la racionalidad económica más allá de sus confines. El sociólogo alemán también recordó que en su planeación teórica basada en la teoría de los sistemas de su maestro Niklas Luhmann, toda teoría sectorial "tiene una presunción de validez universal porque es la única teoría de la sociedad"22. Y los últimos treinta años han sido caracterizados por la tendencia de las diferentes teorías económicas a 'colonizar' el sistema jurídico: "la teoría de los costes de transacción, la teoría de los derechos de propiedad, de la public choice y el análisis económico del derecho son corrientes diferentes de la teoría económica que, en el sistema jurídico, quisieran sustituir el asfíctico concepto de la justicia con el ideal de la eficiencia"23.

A diferencia de otros estudiosos que aclararon cómo el neoliberalismo resistió frente a la crisis económica siempre poniéndose como parámetro de referencia de la acción política $^{24}$, Teubner aún se pregunta si la crisis financiera de 2008 ha ocasionado el derrumbe del monopolio interpretativo de la economía.

De todos modos, los remedios sugeridos por el sociólogo alemán para evitar la verdadera "catástrofe teórica", potencialmente similar a la que, en el pasado, vio sucumbir el derecho frente a la tragedia del totalitarismo político, se hallan en la aplicación de tres condiciones a la relación entre derecho y teoría social: la transversalidad, es decir, el

21. N. Irti, L'ordine giuridico del mercato, Laterza, Roma-Bari, 1998, p. 133.

22. G. Teubner, I rapporti precari tra diritto e teoria sociale, Relación dictada durante la inauguración del año académico del Istituto Italiano per gli Studi Storici, Napoli, 20 de noviembre de 2014, p. 2.

23. Ibid., p. 5.

24. Véanse por ejemplo C. Crouch, The Strange non-death of neo-liberalism, Paperbeck, Cambrige, 2011 y W. Streeck, Gekaufte Zeit:die vertagte Krise des demokratischen Kapitalismus, Suhrkamp, Lipzeig, 2013. 
derecho elige los puntos de contacto entre las diferentes teorías sociales recorriéndolas "transversalmente"; la capacidad de reacción, pues la autonomía de la dogmática jurídica que debe aceptar eventuales formaciones normativas que la sociedad proporciona; la autonormatividad, en la base de la cual el derecho se hace orientar desde un punto de vista normativo solo por los procesos internos al mismo derecho o por la autonormatividad elaborada por las dogmáticas reflexivas de otros sistemas sociales ${ }^{25}$. Por medio de estas tres condiciones, el derecho debería lograr controlar las tendencias expansivas de la racionalidad económica y guardar su identidad.

El enfoque sistémico à la Teubner sigue estando caracterizado por una perspectiva autorreferencial del derecho, producto de la dogmática jurídica que dominaba en Alemania en la segunda mitad del siglo XX.

Para Teubner, siguen siendo los derechos fundamentales los que "limitan las tendencias totalitarias de la matriz política en la sociedad" ${ }^{26}$, mientras que la idea -sacada del constitucionalismo social de David Sciulli ${ }^{27}$ - es que todo sistema social puede crear su propia autónoma "constitución civil", porque "a despecho del derecho de igualdad ratificado por la Constitución, el derecho constitucional justifica tratos desiguales solo si estos quedan justificados por los llamados 'criterios lógicos', conformemente a las ciencias pedagógicas, científicas, médicas, etc." ${ }^{\text {28 }}$.

Desde otra perspectiva teórica y bajo una óptica foucaultiana, vamos a recordar cómo Foucault, en Hay que defender la sociedad, a la hora de elaborar un análisis belicoso, pues no económico ni jurídico del poder, reconoció en el "economicismo" el cruce entre la concepción liberal del poder político (que se identifica más o menos con la jurídica) y la concepción marxista en sentido amplio, porque "la constitución del poder político se hace, entonces, según el modelo de una operación jurídica que sería del orden del intercambio contractual. Analogía manifiesta por consiguiente y que recorre todas estas teorías, entre el poder y los bienes, el poder y la riqueza"29, con la diferencia de que en el primer caso estamos frente a un "poder político que encontraría su modelo formal en el procedimiento del intercambio", mientras que

25. G. Teubner, I rapporti precari tra diritto e teoria sociale, p. 19.

26. G. Teubner, "Ordinamenti frammentati e costituzioni sociali”, en A. Febbrajo, F. Gambino (eds.), Il diritto frammentato, pp. 375-395: 386.

27. Cfr. D. Sciulli, Theory of Societal Constitutionalism. Foundations of a non-Marxist critical theory, Arnold and Caroline Rose Monograph Series of the American Sociological Association, Cambridge University Press, Cambridge-New York-Port Chester-Melbourne-Sidney, 1992.

28. G. Teubner, “Self-subversive Justice: Contingency or Transcendence Formula of Law?”, en, Modern Law Review, vol. 72 , pp. 1-23, 2009.

29. M. Foucault, Hay que defender la sociedad, Curso en el Collège de France (1975-1976), Fondo de Cultura Económica de Argentina, Buenos Aires, 2003, p. 22. 
en el segundo "el poder político tendría en la economía su razón de ser histórica y el principio de su funcionamiento actual"30.

A pesar de que desconociera la utilidad de estos dispositivos teóricos para explicar el ejercicio del poder, igual esto le permitió, en Nacimiento de la biopolítica, mostrar lo importante que había sido la formación de una racionalidad de tipo económico para nuestras maneras de ser sujetos y de constituirnos como tal, y presentar otra genealogía del liberalismo, ya no caracterizado por el reconocimiento jurídico de las libertades o de los derechos 'naturales' de los individuos, sino más bien como un arte de gobierno respetuosa de la 'naturalidad' de los procesos económicos. Así que, también para el filósofo francés el problema de la primacía de la economía en la sociedad se ha hecho realmente concreto con el nacimiento de una forma de gobierno que ha adquirido una función dominante en los dispositivos de reglamentación social, llevando a cabo una continua y progresiva desoberanización de las instituciones. Además, en la clase dictada en el Collège de France del 4 de abril de 1979, Foucault detecta en la construcción de la noción de "sociedad civil" el problema de la presencia por encima del soberano de "algo que se le escapa y no en menor medida, pero ya no son los designios de la Providencia o las leyes de Dios, son los laberintos y meandros del campo económico" ${ }^{31}$. Esto puede realizarse tanto en la versión totalizante de los fisiócratas para los cuales "frente al proceso económico el soberano deberá pasar, para decirlo de algún modo, de la actividad política a la pasividad teórica" por tener que limitarse como si fuera un simple técnico que describe su morfología ${ }^{32}$, como en aquella del neoliberismo para la cual el problema de la sociedad civil se presenta como "la economía jurídica de una gubernamentalidad ajustada a la economía económica” que, tal como explica, luego significa “[...] sociedad civil -que por otra parte no tardará en llamarse sociedad, mientras que a fines del siglo XVIII se la denominaba nación - es lo que va a permitir a una práctica gubernamental y a un arte de gobernar, a una reflexión sobre ese arte de gobernar y por lo tanto a una tecnología gubernamental, una autolimitación que no transgreda ni las leyes de la economía ni los principios del derecho y, tampoco transgreda su exigencia de generalidad gubernamental ni la necesidad de una omnipresencia del gobierno"33.

Así que uno de los cruces entre jurisdicción y veridicción -pues, uno de los fenómenos fundamentales de Occidente y que sigue siendo un elemento de nuestra

\footnotetext{
30. Ibid., p. 23.

31. M. Foucault, Nacimiento de la biopolítica. Curso en el Collège de France (1978-1979), Fondo de Cultura Económica, Buenos Aires, 2007, p. 335.

32. Ibid, p. 333.

33. Ibid.
} 
actualidad- fue la transformación del mercado como objeto de la práctica de gobierno en su verdad. Foucault nos ha enseñado que el liberalismo se ha constituido como un arte del gobierno que realiza y reglamenta aquellos fenómenos de los que la economía política descubre al mismo tiempo la naturaleza, es decir, el mercado, la población, etc. Esto ha permitido que en la esfera política entrara una específica tipología de realidad - pues la 'naturalidad' de los procesos económicos y de su correspondiente subjetivo (el homo oeconomicus), mientras que el mercado se considera el criterio de verdad de la acción de gobierno, el lugar de comprobación y de falsificación para la práctica de gobierno: "Ahora, por el mercado, el gobierno, para poder ser un buen gobierno, deberá actuar en la verdad"34. La economía política no es leal con el gobierno; sin embargo, indicándole al gobierno dónde buscar el principio de verdad de su práctica específica, elimina la práctica de gobierno no jurídica. Además, si en la reconstrucción de Foucault entre finales del siglo XVIII y principios del XIX el derecho se presenta como una forma de limitación externa ${ }^{35}$ al ejercicio del poder, la autoeliminación económica del arte de gobierno liberal -que depende mucho de la utilidad o la inutilidad de la intervención del gobierno- igual debe formularse en la base del derecho, aunque como derecho instrumental que garantice el funcionamiento del orden económico.

Así que, para Foucault, si por un lado el derecho que se define "revolucionario", el que brota de las grandes revoluciones europeas, sigue el camino de la individuación de los derechos originarios y de cómo estos se pueden hacer valer ante cualquier soberano, por otro lado, la economía contribuye a fijar el límite de competencia del gobierno "a través de las fronteras de la utilidad de una intervención gubernamental”"36, y a dar comienzo a la institución del Estado neoliberal.

\section{Consideraciones finales}

La nueva formulación del art. 81 de la Constitución implica el pasaje de una visión procedimental del procedimiento presupuestario, típica de los constituyentes (aunque

34. Ibid.,p. 39.

35. Tal como ha subrayado Michel Senellart, en su análisis del Estado clásico Foucault no ha aceptado nunca la posibilidad de una limitación interna del poder por medio del derecho, porque este funciona "como principio interno de ilimitación del poder (el soberano que no tiene otra finalinad sino la de reforzar su propia soberanía), o bien como principio externo de limitación (la razón de Estado que autoriza a sí misma, en nombre de la necesidad, de la salvación o de la urgencia del Estado, a transgredir las leyes naturales o positivas)" (M. Senellart, "La questione dello stato di diritto in Michel Foucault", en M. Foucault, La strategia dell'accerchiamento.Conversazioni e interventi 1975-1984", editado por S. Vaccaro, Duepunti Edizioni, Palermo, 2009, p. 248).

36. M. Foucault, Nacimiento de la biopolítica, p. 241 ss. 
alguien quería una interpretación más rígida, como el liberal Einaudi), a una visión contenutístico-substancial de la obligación del balance de cuentas que podríamos definir muy normativística ${ }^{37}$. Este planteamiento -que deriva del pacto Euro Plus, aprobado por los Jefes de Estado y de Gobierno de la eurozona el 11 de marzo de 2011, y del llamado Fiscal Compact, firmado en Bruselas el 2 de marzo de 2012 vigente a partir del 1 de enero de 2013- que determina la crisis del decisor estatal (aplastado por la crisis y por la fuerza del mercado), y el rol correctivo de la Corte puesto en práctica por un principio que podríamos seguir definiendo con la expresión 'inviolabilidad de los derechos sociales' (una tesis extrema de hace algunos años) ${ }^{38}$, expresan muy bien la crisis del Welfare State tal como lo conocemos también ahora.

En definitiva, se trataría de una fase de realización efectiva del pasaje "de un sistema en el que prevalece el suministro estatal" a otro "en el cual el Estado interactúa y organiza la intervención de agencias privadas, del socorrismo, de las familias" 39 .

Así que compartimos la tesis del último representante de la Escuela de Frankfurt, Wolfgang Streeck, para el cual la crisis dependería esencialmente de una fallida redistribución de la renta, mientras los capitalistas estarían desmantelando efectivamente el Estado social contra el cual hay que actuar rápido ${ }^{40}$.

De todas formas, cabe destacar que si en la sentencia 10 el principio de la estabilidad financiera fue empleado por la Corte para evitar un resultado injusto e irracional del pronunciamiento de admisión, llevar al extremo su aplicación, incluso alejándose de otros principios institucionales, podría ser peligroso, porque puede influir negativamente en los derechos sociales ${ }^{41}$.

Sin embargo, la sentencia n. 70 de 2015 parece destacar una contratendencia real, desmintiendo pues la reciente idea de Gunther Teubner, para el cual ya debemos afrontar el problema de una injerencia de la racionalidad económica, capaz de sustituir -tal como recordamos antes- el concepto mismo de justicia.

¿Pero estamos seguros de que esta sentencia invalide las tesis teubneriana? Intentemos abandonar la perspectiva funcionalista empleando en cambio la gubernamental, siguiendo algunas intuiciones de Michel Foucault, desde cuyas clases sobre el neolibe-

\footnotetext{
37. También se ha hablado de una verdadera subordinación del equilibrio del balance presupuestario estatal a las fluctuaciones especulativas de las finanzas globalizadas (Cfr. G. Di Gaspare, "L’art. 81 della Costituzione, abdicazione della sovranità finanziaria dello Stato?”, en Amministrazione in cammino, 15 de junio de 2015).

38. D. Bifulco, L'inviolabilità dei diritti sociali, Jovene, Napoli, 2003.

39. C. Pennisi, Istituzioni e cultura giuridica, Giappichelli, Torino, 1998, p. 121.

40. Cfr. W. Streeck, Gekaufte Zeit: die vertagte Krise des demokratischen Kapitalismus.

41. A este propósito, véase también A. Marcheselli, “Dalla Robin tax allo sceriffo di Nottingham”, en Questione Giustizia, 13 de febrero de 2015, http://questionegiustizia.it/articolo/dalla-robin-tax-allo-sceriffo-di-nottingham_13-02-2015.php.
} 
rismo hemos aprendido que el mercado se ha convertido en un lugar de veridicción de las prácticas de gobierno ${ }^{42}$. Ahora sabemos que el primer apartado del art. 81 de la Constitución prevé que "El Estado asegura en su presupuesto el equilibrio entre ingresos y gastos, teniendo en cuenta las fases adversas y las fases favorables del ciclo económico". Aunque en la sentencia 70/2015 los derechos sociales han sido protegidos, a diferencia de otras sentencias en las que la Corte sacralizó de alguna manera el balance presupuestario, ¿es justo decir que esto ocurrió porque la Corte pensó que se hallaba en un ciclo económico favorable? Esto nos induciría claramente a preguntarnos cómo la Corte, en los casos que hemos analizado, establece la negatividad o la positividad de un ciclo económico. Así que, foucaultianamente, podemos preguntarnos qué tipo de saber económico ha adoptado la Corte para tomar sus decisiones entre todas las teorías económicas, tan epistemológicamente frágiles. Si similar hipótesis es admisible, entonces se debería hacer todo lo posible para entender cómo el saber económico se convierte en el objeto de juicio y si la jurisprudencia constitucional está subordinada a este saber y al mercado en general como criterio de veridicción.

Esto no apocaría la interpretación teubneriana antes mencionada con respecto a la subordinación del derecho a la economía, es más la confirmaría. Y eso que sabemos que, para Foucault, el derecho siempre actúa estratégicamente en un ámbito social, y esto no le impide ni contrastar la racionalidad eficientista que está caracterizando a la esfera pública, ni seleccionar entre todas las teorías económicas la más favorable a la ampliación de los derechos y poner otra vez en marcha la exigencia de justicia o nuevas políticas redistributivas.

De todos modos, si las sentencias analizadas varían entre estas dos formas de racionalidad opuestas - por lo menos potencialmente- igual podemos afirmar que la progresiva expansión de las reglas del capitalismo global está transformando radicalmente las relaciones entre derecho y economía. De ahí que sea muy actual lo que Karl Renner dijo a principios del siglo XX: "Law and economics, though appearing to be indissolubly bound together, if considered as statistic at any given moment, yet undergo unequal development in the course of history. Contradictions and contrasts emerge, and their mutual relations are not seldom reversed"43.

42. "El mercado debe decir la verdad, debe decir la verdad con respecto a la práctica gubernamental. En lo sucesivo, y de una manera simplemente secundaria, será su papel de veridicción el que rija, dicte, prescriba los mecanismos jurisdiccionales a la ausencia de mecanismos juridiccionales con los cuales deberá articularse", M. Foucault, Nacimiento de la biopolitica, p. 50 .

43. K. Renner, The Institutions of Private Law and their Social Function (1929), Routledge, New York, 2001, p. 251. 